\title{
Shooting methods and topological transversality
}

\author{
B. Buffoni \\ Département de mathématiques, Ecole Polytechnique Fédérale de \\ Lausanne, 1015 Lausanne, Switzerland (Boris.Buffoni@epfl.ch) \\ (MS received 16 April 1997; accepted 23 October 1998)
}

\begin{abstract}
We show that shooting methods for homoclinic or heteroclinic orbits in dynamical systems may automatically guarantee the topological transversality of the stable and unstable manifolds. The interest of such results is twofold. First, these orbits persist under perturbations which destroy the structure allowing the shooting method and, second, topological transversality is often sufficient when some kind of transversality is required to obtain chaotic dynamics. We shall focus on heteroclinic solutions in the extended Fisher-Kolmogorov equation.
\end{abstract}

\section{Introduction}

Shooting methods are quite efficient to study numerically homoclinic and heteroclinic solutions of ordinary differential equations (ODEs) (see, for example, [8]). Often, some kind of transversality of the stable and unstable manifolds is required to prove that a homoclinic or heteroclinic orbit leads to chaotic behaviour. However, as observed by Mischaikov and Mrozek [25], estimates on the derivative along the solution are needed to establish transversality and they may demand a considerable amount of computing resources. Mischaikov and Mrozek introduce in [24-26] a topological condition that is weaker than transversality and more amenable to computation. Their theory is based on the Conley index and is applied to the Lorenz equation. The use of the Conley index to study chaotic dynamics was thoroughly investigated in the work by Easton [15] and in the three papers by Churchill and $\operatorname{Rod}[10-12]$.

Alternatively, for some special ODEs, a shooting method has been developed that not only proves the existence of a connecting orbit, but also gives an infinite family of chaotic solutions. A precise and complete description of the dynamics of those ODEs is possible in this way, and many physically relevant equations have been successfully investigated (see, for example, $[3,5,9,19,29-31,33]$ ).

For Hamiltonian and Lagrangian systems, the calculus of variations has been the main ingredient of a third approach, which has raised a lot of interest in recent years (see, for example, $[1,2,13,32]$ ). Here again, a first connecting orbit is found and a much weaker assumption than transversality has been proved to be sufficient in many cases for the system to be chaotic. Some results are available for partial differential equations (PDEs) [13].

Thanks to collaboration with A. R. Champneys, J. F. Toland and É. Séré on the following fourth-order equation occurring in water-wave theory:

$$
u^{(\mathrm{iv})}(x)+P u^{\prime \prime}(x)+u(x)-u^{2}(x)=0, \quad x \in \mathbb{R},
$$


the author got acquainted with the shooting method and the variational approach [4-7]. The aim of this paper is to show that shooting methods ensure a weak form of transversality, called here 'topological transversality', which is defined in the appendix.

In $\S 2$, we relax the classical transversality condition in the Smale-Birkhoff theorem for discrete dynamical systems and, in $\S 3$, in the theory of Devaney for autonomous fourth-order Hamiltonian systems with a saddle-focus equilibrium. In both cases, we show that a topological transversality assumption is sufficient to ensure the dynamics to be chaotic, without requiring the analyticity of the dynamical system. Although we only deal with homoclinic orbits, our results extend to closed paths of heteroclinic orbits without additional difficulties. The statements can be considered as special cases of those of Easton [15], but the proofs are closely related to the technique of Palmer presented in [27] and to the variational construction of multibump homoclinic solutions (see, for example, [4,32]).

Instead of equation (1.1), we shall focus in $\S \S 4-6$ on the extended Fisher-Kolmogorov equation

$$
-\gamma u^{(\mathrm{iv})}(x)+u^{\prime \prime}(x)+u(x)-u^{3}(x)=0, \quad x \in \mathbb{R},
$$

which has been analysed by shooting methods by Peletier and Troy [29-31] and by variational methods by Kalies et al. [22,23]. In contrast with (1.1), where homoclinic solutions to 0 describe travelling waves, the objects of consideration in (EFK) are heteroclinic solutions (also called kinks) connecting the equilibria $u \equiv-1$ and $u \equiv 1$. Heteroclinic orbits being easier to deal with in the present context, this is why (EFK), rather than equation (1.1), is chosen as an example to illustrate the ideas of this paper. The existence of a kink for (EFK) has been established in $[29,30]$.

In $\S 5$, we show the existence of a kink for (EFK) when $0<\gamma \leqslant \frac{1}{8}$, that is, the topologically transverse intersection of the stable and unstable manifolds. The techniques used stem from the work of Hofer and Toland [21] and they are reminiscent of the index for monotonic Hamiltonian systems devised by Toland in [34]. Since the completion of this work, Van den Berg has proved uniqueness and transversality [35].

In $\S 6$, which can be read independently of $\S 5$, we consider (EFK) in the case $\gamma>\frac{1}{8}$ and deduce the known fact $[22,23,31]$ that the extended Fisher-Kolmogorov equation is chaotic for this range of values of the parameter $\gamma$. The proofs can be adapted to equation (1.1) and partly to higher-dimensional problems of the kind introduced in [21].

\section{The Smale-Birkhoff homoclinic theorem}

This theorem reads as follows (version taken from [17]).

THEOREM 2.1. Let $f: \mathbb{R}^{n} \rightarrow \mathbb{R}^{n}$ be a diffeomorphism such that $p$ is a hyperbolic fixed point and there exists a point $q \neq p$ of (algebraic) transversal intersection between $W^{\mathrm{s}}(p)$ and $W^{\mathrm{u}}(p)$. Then $f$ has a hyperbolic invariant set on which $f$ is topologically equivalent to a subshift of finite type. 
$W^{\mathrm{s}}(p)$ and $W^{\mathrm{u}}(p)$ denote, respectively, the stable and unstable manifolds of $p$. Instead of algebraic transversality, we now suppose topological transversality. See the appendix for the meaning of 'compact piece of manifold' and for the definition of the topological intersection number $I\left(M_{1}, M_{2}\right)$ of two compact pieces of manifolds $M_{1}$ and $M_{2}$.

THEOREM 2.2. Let $f: \mathbb{R}^{n} \rightarrow \mathbb{R}^{n}$ be a diffeomorphism such that $p$ is a hyperbolic fixed point, and there exists a compact piece $M_{\mathrm{u}}$ of $W^{\mathrm{u}}(p)$ and a compact piece $M_{\mathrm{s}}$ of $W^{\mathrm{s}}(p)$ such that $p \notin N:=M_{\mathrm{u}} \cap M_{\mathrm{s}}, N \subset \operatorname{int}\left(M_{\mathrm{u}}\right) \cap \operatorname{int}\left(M_{\mathrm{s}}\right)$ and $I\left(M_{\mathrm{u}}, M_{\mathrm{s}}\right) \neq 0$. Then there exists a bounded neighbourhood $U_{N} \subset \mathbb{R}^{n}$ of $N$, a small neighbourhood $U_{p}$ of $p$ whose closure does not intersect $\bar{U}_{N}$, and an integer $\tilde{m} \geqslant 2$ such that for each choice of $k \geqslant 1$ and

$$
\left(m_{1}, \ldots, m_{k}\right) \in\{m \in \mathbb{N}: m \geqslant \tilde{m}\}^{k},
$$

(i) (homoclinic orbits) there exists $\left(q_{1}, \ldots, q_{k+1}\right) \in U_{N}^{k+1}$, with $q_{1} \in M_{\mathrm{u}}, q_{i+1}=$ $f^{m_{i}}\left(q_{i}\right)$ for $1 \leqslant i \leqslant k, q_{k+1} \in M_{\mathrm{s}}$ and $f^{[i / 2]}\left(q_{i}\right) \in U_{p}$ for $1 \leqslant i \leqslant k$;

(ii) (periodic orbits) there exists $\left(q_{1}, \ldots, q_{k+1}\right) \in U_{N}^{k+1}$, with $q_{i+1}=f^{m_{i}}\left(q_{i}\right)$ for $1 \leqslant i \leqslant k, q_{k+1}=q_{1}$ and $f^{[i / 2]}\left(q_{i}\right) \in U_{p}$ for $1 \leqslant i \leqslant k$.

Moreover, for each bi-infinite sequence $\left(m_{i}, i \in \mathbb{Z}\right) \subset\{m \in \mathbb{N}: m \geqslant \tilde{m}\}$, there exists $\left(q_{i}, i \in \mathbb{Z}\right) \subset \bar{U}_{N}$ with $q_{i+1}=f^{m_{i}}\left(q_{i}\right)$ and $f^{[i / 2]}\left(q_{i}\right) \in \bar{U}_{p}$ for all $i \in \mathbb{Z}$.

Note that $N$ can be a very complicated set. This result yields a rich family of homoclinic and periodic orbits, as well as an uncountable set of wild orbits. In particular, there exists a compact invariant set on which $f$ has positive topological entropy [17, defintion 5.8.3]. This theorem does not ensure any uniqueness result. For example, there may be many homoclinic orbits corresponding to a given sequence $\left(m_{1}, \ldots, m_{k}\right)$.

Proof. For $\tilde{m}$ sufficiently large and each $m \geqslant \tilde{m}$, the $\lambda$ lemma [28] gives an embed$\operatorname{ding} \lambda_{m} \in C^{1}\left(M_{\mathrm{s}} \times M_{\mathrm{u}}, \mathbb{R}^{2 n}\right)$ such that

(i) if $(a, b) \in \Lambda_{m}:=\operatorname{Image}\left(\lambda_{m}\right)$, then $b=f^{m}(a)$ and $f^{[m / 2]}(a) \in U_{p}$;

(ii) $\lim _{m \rightarrow \infty} \sup \left\{\operatorname{dist}\left(c, \lambda_{m}(c)\right): c \in M_{\mathrm{s}} \times M_{\mathrm{u}}\right\}=0$.

Let us give more details on how the mapping $\lambda_{m}$ is obtained. We introduce two $C^{1}$ families of discs $\left(D_{a}^{\mathrm{u}}, a \in M_{\mathrm{s}}\right)$ and $\left(D_{b}^{\mathrm{s}}, b \in M_{\mathrm{u}}\right)$ transversal, respectively, to $M_{\mathrm{s}}$ and $M_{\mathrm{u}}$ and such that no disc meets another disc of the same family. By the Hartman-Grobman theorem and the $\lambda$ lemma $[28], f^{[m / 2]}\left(D_{a}^{\mathrm{u}}\right)$ and $f^{[m / 2]-m}\left(D_{b}^{\mathrm{s}}\right)$ intersect each other transversally near $p$ for large $m$ at a unique point $q(a, b)$ that belongs to the same component of $f^{[m / 2]}\left(D_{a}^{\mathrm{u}}\right) \cap U_{p}$ as $f^{[m / 2]}(a)$ and to the same component of $f^{[m / 2]-m}\left(D_{b}^{\mathrm{s}}\right) \cap U_{p}$ as $f^{[m / 2]-m}(b)$. By compactness of $M_{\mathrm{s}}$ and $M_{\mathrm{u}}$, this can be done uniformly in $(a, b) \in M_{\mathrm{s}} \times M_{\mathrm{u}}$. We set

$$
\lambda_{m}(a, b)=\left(f^{-[m / 2]}(q(a, b)), f^{m-[m / 2]}(q(a, b))\right) .
$$

To prove the existence of the rich family of orbits, choose any

$$
\left(m_{1}, \ldots, m_{k}\right) \in\{m \in \mathbb{N}: m \geqslant \tilde{m}\}^{k} .
$$


First suppose that $k=2 l$ for some positive integer $l$. Homoclinic orbits corresponding to $\left(m_{1}, \ldots, m_{k}\right)$ are given by the points in the intersection of

$$
\Lambda_{m_{1}} \times \cdots \times \Lambda_{m_{2 i-1}} \times \cdots \times \Lambda_{m_{2 l-1}} \times M_{\mathrm{s}}
$$

and

$$
M_{\mathrm{u}} \times \Lambda_{m_{2}} \times \cdots \times \Lambda_{m_{2 i}} \times \cdots \times \Lambda_{m_{2 l}}
$$

in $\mathbb{R}^{(k+1) n}$. For $\tilde{m}$ large, these sets are admissible for intersection theory and a homotopy gives that their intersection number is equal to

$$
\begin{aligned}
I\left(\left(M_{\mathrm{s}} \times M_{\mathrm{u}}\right) \times \cdots \times\left(M_{\mathrm{s}} \times M_{\mathrm{u}}\right) \times M_{\mathrm{s}}, M_{\mathrm{u}} \times\left(M_{\mathrm{s}} \times M_{\mathrm{u}}\right) \times \cdots \times\left(M_{\mathrm{s}} \times M_{\mathrm{u}}\right)\right) & \\
& = \pm I\left(M_{\mathrm{u}}, M_{\mathrm{s}}\right)^{k+1} \neq 0 .
\end{aligned}
$$

Periodic orbits are obtained in the same way by considering the intersection of

$$
\sigma_{k}\left(\Lambda_{m_{1}} \times \cdots \times \Lambda_{m_{2 i-1}} \times \cdots \times \Lambda_{m_{2 l-1}}\right)
$$

and

$$
\Lambda_{m_{2}} \times \cdots \times \Lambda_{m_{2 i}} \times \cdots \times \Lambda_{m_{2 l}},
$$

where $\sigma_{k}$ is the permutation $\sigma_{k}\left(q_{1}, \ldots, q_{k}\right)=\left(q_{2}, \ldots, q_{k}, q_{1}\right)$, with $q_{i} \in \mathbb{R}^{n}$ for all $i$.

Second suppose that $k=2 l+1$ for some non-negative integer $l$. Homoclinic orbits corresponding to $\left(m_{1}, \ldots, m_{k}\right)$ are given by the points in the intersection of

$$
\Lambda_{m_{1}} \times \cdots \times \Lambda_{m_{2 i+1}} \times \cdots \times \Lambda_{m_{2 l+1}}
$$

and

$$
M_{\mathrm{u}} \times \Lambda_{m_{2}} \times \cdots \times \Lambda_{m_{2 i}} \times \cdots \times \Lambda_{m_{2 l}} \times M_{\mathrm{s}}
$$

in $\mathbb{R}^{(k+1) n}$. For $\tilde{m}$ large, these sets are admissible for intersection theory and a homotopy gives that their intersection number is equal to

$$
\begin{array}{r}
I\left(\left(M_{\mathrm{s}} \times M_{\mathrm{u}}\right) \times \cdots \times\left(M_{\mathrm{s}} \times M_{\mathrm{u}}\right), M_{\mathrm{u}} \times\left(M_{\mathrm{s}} \times M_{\mathrm{u}}\right) \times \cdots \times\left(M_{\mathrm{s}} \times M_{\mathrm{u}}\right) \times M_{\mathrm{s}}\right) \\
= \pm I\left(M_{\mathrm{u}}, M_{\mathrm{s}}\right)^{k+1} \neq 0 .
\end{array}
$$

Periodic orbits are obtained by considering the intersection of

$$
\sigma_{k+1}\left(\Lambda_{m_{1}} \times \cdots \times \Lambda_{m_{2 i+1}} \times \cdots \times \Lambda_{m_{2 l+1}}\right)
$$

and

$$
\Lambda_{m_{2}} \times \cdots \times \Lambda_{m_{2 i}} \times \cdots \times \Lambda_{m_{2 l}} \times \Delta
$$

where $\Delta:=\left\{(q, q): q \in \mathbb{R}^{n}\right\}$ is the diagonal in $\left(\mathbb{R}^{n}\right)^{2}$.

The final assertion of the theorem is proved by taking limits of homoclinic orbits, as follows. Consider a bi-infinite sequence $\left(m_{i}, i \in \mathbb{Z}\right) \subset\{m \in \mathbb{N}: m \geqslant \tilde{m}\}$. For $r \geqslant 1$ and $\left(m_{-r}, \ldots, m_{r-1}\right)$, let

$$
\left(q_{-r}^{(r)}, \ldots, q_{r}^{(r)}\right) \in U_{N}^{2 r+1}
$$

be given by the first part of the theorem. Considering a subsequence, if necessary, the limit

$$
q_{0}:=\lim _{r \rightarrow \infty} q_{0}^{(r)}
$$


exists. Set

$$
q_{i}:=f^{m_{i}}\left(q_{0}\right)=\lim _{r \rightarrow \infty} q_{i}^{(r)}
$$

for $i \in \mathbb{Z}$.

\section{Devaney's theorem}

We consider the Hamiltonian system

$$
z^{\prime}=J \nabla H(z), \quad z \in \mathbb{R}^{4},
$$

where $J$ is the standard symplectic matrix and $H \in C^{5}\left(\mathbb{R}^{4} ; \mathbb{R}\right)$. We suppose that $H(0)=0, \nabla H(0)=0$ and $0 \in \mathbb{R}^{4}$ is a saddle-focus, i.e. the spectrum of $J H^{\prime \prime}(0)$ consists of $\pm \lambda \pm \mathrm{i} \omega$ for some positive $\lambda$ and $\omega$. The following result is due to Devaney [14].

THEOREM 3.1. If $H$ is analytic and $W^{\mathrm{u}}(0)$ and $W^{\mathrm{s}}(0)$ intersect each other transversally in $H_{0}:=\left\{z \in \mathbb{R}^{4}: H(z)=0\right\}$ along a (non-trivial) homoclinic orbit, then a Smale horseshoe is 'contained' in the dynamics.

Again, our aim is to weaken the transversality assumption. It is known that the analyticity of $H$ is not necessary [36] and we shall only require $H$ to be of class $C^{5}$.

We suppose that some bounded open set $0 \notin U \subset \mathbb{R}^{4}$ admits a parametrization $\psi: Y \times I_{1} \times I_{2} \rightarrow U$ such that

(i) $I_{1}$ and $I_{2}$ are open intervals containing 0 ;

(ii) $Y$ is an orientable $C^{1}$ manifold of dimension two;

(iii) $\psi$ is a diffeomorphism;

(iv) $(H \circ \psi)(y, h, s)=h$ on $Y \times I_{1} \times I_{2}$;

(v) under the flow, $\mathrm{d} s / \mathrm{d} t>0$ on $U$;

(vi) there exist two compact pieces of manifolds, $M_{\mathrm{u}}$ and $M_{\mathrm{s}}$, that are connected, included in $Y$, of dimension one, admissible for intersection theory and such that $M_{\mathrm{s}} \times M_{\mathrm{u}}$ is simply connected;

(vii) $\left\{\psi(y, 0,0): y \in M_{\mathrm{u} / \mathrm{s}}\right\} \subset W^{\mathrm{u} / \mathrm{s}}(0)$ and $I\left(M_{\mathrm{u}}, M_{\mathrm{s}}\right) \neq 0$.

The parameter $s$ describes a family of Poincaré sections of the flow. In what follows, we shall restrict attention to the Poincaré section $\{\psi(y, h, s): s=0\}$. Moreover, $t \rightarrow \Gamma(t, y, h)$ will denote the solution of (3.1) starting at $\psi(y, h, 0)$ at time $t=0$, defined on some maximal open time interval containing $t=0$.

THEOREM 3.2. There exists a constant $\tau_{0}$ and a small neighbourhood $V \subset \mathbb{R}^{4}$ of 0 whose closure does not intersect $\bar{U}$, such that for each choice of $k \in \mathbb{N}$ and $\left(m_{1}, \ldots, m_{k}\right) \in \mathbb{N}^{k}$, 
(i) (homoclinic orbits) there exists $\left(q_{1}, \ldots, q_{k+1}\right) \in Y^{k+1}$ with $q_{1} \in M_{\mathbf{u}}, q_{k+1} \in$ $M_{\mathrm{s}}$ and, for all $1 \leqslant i \leqslant k$,

$$
\psi\left(q_{i+1}, 0,0\right)=\Gamma\left(T_{i}, q_{i}, 0\right) \quad \text { and } \quad \Gamma\left(\frac{1}{2} T_{i}, q_{i}, 0\right) \in V
$$

for some $T_{i} \in \mathbb{R}$ satisfying

$$
T_{i} \in\left[\tau_{0}+\left(m_{i}-\frac{1}{2}\right) \pi / \omega, \tau_{0}+\left(m_{i}+\frac{9}{2}\right) \pi / \omega\right]
$$

(ii) (periodic orbits) there exists $\left(q_{1}, \ldots, q_{k+1}\right) \in Y^{k+1}$ such that $q_{1}=q_{k+1}$ and relation (3.2) holds for all $1 \leqslant i \leqslant k$.

Moreover, for each bi-infinite sequence $\left(m_{i}, i \in \mathbb{Z}\right) \subset \mathbb{N}$, there exists a sequence $\left(q_{i}, i \in \mathbb{Z}\right) \subset Y$ such that relation (3.2) holds for all $i \in \mathbb{Z}$.

Finally, for each of these three families, all orbits are distinct.

Proof. For $T_{0}$ sufficiently large, the $\lambda$ lemma for continuous dynamical systems [28, ch. 2, lemma 7.2] gives a $C^{1}$ map $\left.\lambda:\right] T_{0}, \infty\left[\times M_{\mathrm{s}} \times M_{\mathrm{u}} \rightarrow Y^{2} \times I_{1}\right.$ such that

(i) if $(a, b, h) \in \operatorname{Image}(\lambda(T, .)$,$) , then \Gamma(T, a, h)=\psi(b, h, 0)$;

(ii) $\lim _{T \rightarrow \infty} \sup \left\{\operatorname{dist}(\lambda(T, c),(c, 0)): c \in M_{\mathrm{s}} \times M_{\mathrm{u}}\right\}=0$.

As in the previous section, the map $\lambda$ is obtained by introducing two $C^{1}$ families of two-dimensional discs in $Y \times I_{1},\left(D_{a}^{\mathrm{u}}, a \in M_{\mathrm{s}}\right)$ and $\left(D_{b}^{\mathrm{s}}, b \in M_{\mathrm{u}}\right)$, transversal respectively to $M_{\mathrm{s}} \times\{0\}$ and $M_{\mathrm{u}} \times\{0\}$ and such that no disc meets another disc of the same family. By the $\lambda$ lemma, $\Gamma\left(T / 2, D_{a}^{\mathrm{u}}\right)$ and $\Gamma\left(-T / 2, D_{b}^{\mathrm{s}}\right)$ intersect each other transversally near 0 for large $T>0$ at some point $q(a, b) \in \mathbb{R}^{4}$ which belongs to the same component of $\Gamma\left(\frac{1}{2} T, D_{a}^{\mathrm{u}}\right) \cap V$ as $\Gamma\left(\frac{1}{2} T, a, 0\right)$ and to the same component of $\Gamma\left(-\frac{1}{2} T, D_{b}^{\mathrm{s}}\right) \cap V$ as $\Gamma\left(-\frac{1}{2} T, b, 0\right)$. By compactness of $M_{\mathrm{s}}$ and $M_{\mathrm{u}}$, this can be done uniformly in $(a, b) \in M_{\mathrm{s}} \times M_{\mathrm{u}}$. Then $\lambda(T, a, b)=(c, d, h)$ if and only if (by definition) $\Gamma\left(\frac{1}{2} T, c, h\right)=q(a, b)=\Gamma\left(-\frac{1}{2} T, d, h\right)$.

Set $\mathcal{H}(a, h):=h$ for $a \in Y^{2}$. It is proved in [4, lemma 11] that $(\mathcal{H} \circ \lambda)(T, a)$ oscillates around 0 as a function of $T \gg 0$, for fixed $a \in M_{\mathrm{s}} \times M_{\mathrm{u}}$, roughly like

$$
\exp (-\lambda T) \sin (\omega T-\omega T(a))
$$

for some shift $\tau(a) \in \mathbb{R}$ continuous in $a \in M_{\mathrm{s}} \times M_{\mathrm{u}}$. A priori, the function $\tau$ is multivalued, but we can select a continuous branch defined over $M_{\mathrm{s}} \times M_{\mathrm{u}}$ because $M_{\mathrm{s}} \times M_{\mathrm{u}}$ is supposed to be simply connected.

Note that

$$
\inf \left\{\exp (\lambda T) \sin (\omega T-\omega \tau(a)) \mathcal{H}(\lambda(T, a)): a \in M_{\mathrm{s}} \times M_{\mathrm{u}}\right\}>0
$$

for all $T>0$ such that $\omega T-\omega \tau(a) \in \frac{1}{2} \pi+\pi Z$. Clearly, by adding some constant to the shift-function $\tau$, we can assume, without loss of the generality, that $\tau(a) \in] T_{0}, \infty\left[\right.$ for all $a \in M_{\mathrm{S}} \times M_{\mathrm{u}}$ and any given $T_{0}$. As a consequence, for all $\sigma \in \pi /(2 \omega)+(\pi / \omega) \mathbb{Z}_{+}$,

$$
\inf \left\{\sin (\omega \sigma) \operatorname{sgn}(\mathcal{H}(\lambda(\tau(a)+\sigma, a))): a \in M_{\mathrm{s}} \times M_{\mathrm{u}}\right\}>0
$$


if $T_{0}>0$ is large enough. For $m \in \mathbb{N}$, we set

$$
\begin{aligned}
\Lambda_{m}=\left\{(c, h, d, 0) \in\left(Y \times I_{1}\right)^{2}:(c, d, h)=\lambda(\tau(a)+\sigma, a),\right. & \\
& \left.\quad a \in M_{\mathrm{u}} \times M_{\mathrm{s}},|\sigma-m \pi / \omega| \leqslant \pi /(2 \omega)\right\} .
\end{aligned}
$$

If $T_{0}$ is large enough, $\Lambda_{m}$ is a compact piece of a $C^{1}$ submanifold of dimension three. Note that the function $\tau$ and the constant $\tau_{0}$ appearing in the statement of the theorem are related by $\tau_{0}=\min \left\{\tau(a): a \in M_{\mathrm{s}} \times M_{\mathrm{u}}\right\}$ and that

$$
\max \left\{\tau(a): a \in M_{\mathrm{s}} \times M_{\mathrm{u}}\right\}<\tau_{0}+\frac{4 \pi}{\omega} .
$$

Choose $\left(m_{1}, \ldots, m_{k}\right) \in \mathbb{N}^{k}$. First suppose that $k=2 l$ for some positive integer $l$. Homoclinic orbits corresponding to $\left(m_{1}, \ldots, m_{k}\right)$ are given by the points in the intersection of

$$
\Lambda_{m_{1}} \times \cdots \times \Lambda_{m_{2 i-1}} \times \cdots \times \Lambda_{m_{2 l-1}} \times\left(M_{\mathrm{s}} \times[-1,1]\right)
$$

and

$$
\left(M_{1} \times\{0\}\right) \times \Lambda_{m_{2}} \times \cdots \times \Lambda_{m_{2 i}} \times \cdots \times \Lambda_{m_{2 l}}
$$

in $\left(Y \times I_{1}\right)^{k+1}$. These sets are admissible for intersection theory and a homotopy gives that their intersection number is equal to the one of

$$
\left(M_{\mathrm{s}} \times[-1,1] \times M_{\mathrm{u}} \times\{0\}\right) \times \cdots \times\left(M_{\mathrm{s}} \times[-1,1] \times M_{\mathrm{u}} \times\{0\}\right) \times\left(M_{\mathrm{s}} \times[-1,1]\right)
$$

and

$$
\left(M_{\mathrm{u}} \times\{0\}\right) \times\left(M_{\mathrm{s}} \times[-1,1] \times M_{\mathrm{u}} \times\{0\}\right) \times \cdots \times\left(M_{\mathrm{s}} \times[-1,1] \times M_{\mathrm{u}} \times\{0\}\right)
$$

which is equal to $\pm I\left(M_{\mathrm{u}}, M_{\mathrm{s}}\right)^{k+1} \neq 0$.

For $k$ odd, for periodic orbits and for other wild orbits, the method is the same; see the preceding section.

\section{The EFK equation}

The extended Fisher-Kolmogorov equation (EFK) is given by

$$
-\gamma u^{(\mathrm{iv})}(t)+u^{\prime \prime}(t)+u(t)-u^{3}(t)=0,
$$

where $\gamma>0$ is a parameter. Setting

$$
V\left(u, u^{\prime \prime}\right)=\frac{1}{4}\left(u^{2}-1\right)^{2}-\left(\frac{1}{2} \gamma\right)\left(u^{\prime \prime}\right)^{2}
$$

and

$$
T=\left(\begin{array}{cc}
-1 & \gamma \\
\gamma & 0
\end{array}\right),
$$

it may be written as the Lagrangian system

$$
T q^{\prime \prime}+\nabla V(q)=0,
$$


with $q=\left(u, u^{\prime \prime}\right)$. This Lagrangian system belongs to the family studied in [21]. Its corresponding Hamiltonian is

$$
H\left(q, q^{\prime}\right)=\frac{1}{2}\left\langle T q^{\prime}, q^{\prime}\right\rangle+V(q)
$$

Equation (EFK) admits three equilibria: $u \equiv-1, u \equiv 0$ and $u \equiv 1$. We shall be interested in heteroclinic connections (also called kinks) between -1 and 1 . For such solutions, the Hamiltonian takes the value 0 and hence $H_{0}:=\left\{z \in \mathbb{R}^{4}: H(z)=0\right\}$ will play a particular role, as well as $V_{0}:=\left\{q \in \mathbb{R}^{2}: V(q)=0\right\}$.

The extended Fisher-Kolmogorov equation enjoys many symmetries. If $(q(t)$, $\left.q^{\prime}(t)\right)$ is an orbit, then so are $\left(q(-t),-q^{\prime}(-t)\right),\left(-q(t),-q^{\prime}(t)\right)$ and $\left(-q(-t), q^{\prime}(-t)\right)$. These symmetries are sometimes used in a essential way and sometimes only to keep at a low level the amount of notations or to shorten an argument. For example, the stable and unstable manifolds of the equilibrium $u \equiv-1$ and those of the equilibrium $u \equiv 1$ are related by the previous symmetries, which allows us to halve the amount of notations.

For any orbit $\left(q, q^{\prime}\right)$ lying in $H_{0}$, i.e. $\frac{1}{2}\left\langle T q^{\prime}, q^{\prime}\right\rangle+V(q)=0$, the sign of $\left\langle T q^{\prime}, q^{\prime}\right\rangle$ changes only when $q$ crosses the set $V_{0}$. Hence the vector $q^{\prime}$ stays in one of the following open cones, as long as $q$ remains away from $V_{0}$ :

$$
\begin{aligned}
& K_{0}=\left\{v \in \mathbb{R}^{2}:\langle T v, v\rangle<0, v_{1}>0\right\}=\left\{v \in \mathbb{R}^{2}:-v_{1}+2 \gamma v_{2}<0, v_{1}>0\right\}, \\
& K_{1}=\left\{v \in \mathbb{R}^{2}:\langle T v, v\rangle>0, v_{1}>0\right\}=\left\{v \in \mathbb{R}^{2}:-v_{1}+2 \gamma v_{2}>0, v_{1}>0\right\}, \\
& K_{2}=\left\{v \in \mathbb{R}^{2}:\langle T v, v\rangle<0, v_{1}<0\right\}=\left\{v \in \mathbb{R}^{2}:-v_{1}+2 \gamma v_{2}>0, v_{1}<0\right\}, \\
& K_{3}=\left\{v \in \mathbb{R}^{2}:\langle T v, v\rangle>0, v_{1}<0\right\}=\left\{v \in \mathbb{R}^{2}:-v_{1}+2 \gamma v_{2}<0, v_{1}<0\right\} .
\end{aligned}
$$

More precisely, if $V(q)>0$, then $q^{\prime}$ stays in $K_{0} \cup K_{2}$, and if $V(q)<0$, then $q^{\prime}$ stays in $K_{1} \cup K_{3}$. As a consequence, the path $q(t)$ of an orbit $\left(q(t), q^{\prime}(t)\right)$ in $H_{0}$ is monotonic as long as $q$ does not meet $V_{0}$. This remark, due in a more general setting to Hofer and Toland [21], is the basic ingredient of their shooting method. Note that the slope of $\partial K_{0} \cap \partial K_{1}$ is $1 /(2 \gamma)$ and that $\partial K_{0} \cap \partial K_{3}$ is vertical. The following lemma describes the set $V_{0}$.

LEMMA 4.1. $V_{0} \subset \mathbb{R}^{2}$ is the union of two $C^{\infty}$ curves intersecting each other at $(-1,0)$ and $(1,0)$. The derivatives of these curves at the intersection points are $\pm \sqrt{2 / \gamma}$. The set

$$
\mathcal{C}:=\left\{q \in \mathbb{R}^{2}: V(q)>0,-1<q_{1}<1\right\}
$$

is strictly convex. Finally, if $0<\gamma \leqslant \frac{1}{8}$, then

$$
\mathcal{C} \subset\left((-1,0)+K_{0}\right) \cap\left((1,0)+K_{2}\right)
$$

Proof. From $V\left(q_{1}, q_{2}\right)=0$, we find $q_{2}= \pm(2 \gamma)^{-1 / 2}\left(q_{1}^{2}-1\right)$. We deduce that $V_{0}$ is the union of two smooth curves which intersect each other at $( \pm 1,0)$. It is also easy to compute their slopes at their intersection points. Let us show that $\left\langle V^{\prime \prime} \nabla V^{\perp}, \nabla V^{\perp}\right\rangle<0$ at every $q \in \partial \mathcal{C} \backslash\{(1,0),(-1,0)\}$. We have

$$
\nabla V(q)=\left(q_{1}\left(q_{1}^{2}-1\right),-\gamma q_{2}\right) \text { and } \nabla V(q)^{\perp}=\left(\gamma q_{2}, q_{1}\left(q_{1}^{2}-1\right)\right)
$$


Hence

$$
\begin{aligned}
\left\langle V^{\prime \prime} \nabla V^{\perp}, \nabla V^{\perp}\right\rangle & =\left\langle\left(\begin{array}{cc}
3 q_{1}^{2}-1 & 0 \\
0 & -\gamma
\end{array}\right)\left(\begin{array}{c}
q_{2} \gamma \\
q_{1}\left(q_{1}^{2}-1\right)
\end{array}\right),\left(\begin{array}{c}
q_{2} \gamma \\
q_{1}\left(q_{1}^{2}-1\right)
\end{array}\right)\right\rangle \\
& =\gamma^{2}\left(3 q_{1}^{2}-1\right) q_{2}^{2}-\gamma q_{1}^{2}\left(q_{1}^{2}-1\right)^{2} \\
& =\gamma^{2}\left(3 q_{1}^{2}-1\right) q_{2}^{2}-2 \gamma^{2} q_{1}^{2} q_{2}^{2} \\
& =\left(q_{1}^{2}-1\right) \gamma^{2} q_{2}^{2}<0 .
\end{aligned}
$$

Setting $u=-1+w,($ EFK) becomes

$$
-\gamma w^{(\mathrm{iv})}(t)+w^{\prime \prime}(t)+w(t)(w(t)-1)(2-w(t))=0
$$

the linearization of which is $-\gamma w^{(\mathrm{iv})}(t)+w^{\prime \prime}(t)-2 w(t)=0$. Solutions of the form $w(t)=\exp \lambda t$ are given by

$$
\lambda^{2}=\frac{1 \pm \sqrt{1-8 \gamma}}{2 \gamma} .
$$

We deduce that $u \equiv-1$ is a real saddle if $0<\gamma \leqslant \frac{1}{8}$ and a saddle-focus if $\gamma>\frac{1}{8}$. We denote by $W^{\mathrm{u}}(-1)$ and $W_{\text {loc }}^{\mathrm{u}}(-1)$ its global and local unstable manifolds. By symmetry, we obtain the same result for $u \equiv 1$ and we denote by $W^{\mathrm{s}}(1)$ and $W_{\mathrm{loc}}^{\mathrm{s}}(1)$ its global and local stable manifolds.

\section{The case $\gamma \leqslant \frac{1}{8}$}

Throughout this section, we assume that $0<\gamma \leqslant \frac{1}{8}$. As a consequence of the monotonicity property, if $\left(q(t), q^{\prime}(t)\right)$ is an orbit in $H_{0}$ such that $q(0) \in \mathcal{C}$ and $q^{\prime}(0) \in K_{0}$, then either $\lim _{t \rightarrow+\infty} q(t)=(1,0)$ or $q(t)$ has to leave $\mathcal{C}$ at some positive exit time $\tau^{+}\left(q(0), q^{\prime}(0)\right)$ continuous in $\left(q(0), q^{\prime}(0)\right)$. In the second case, $q^{\prime}\left(\tau^{+}\right) \neq 0$ belongs to $\partial K_{0} \cap \partial K_{1}$ or $\partial K_{0} \cap \partial K_{3}$ if, respectively, $q_{2}\left(\tau^{+}\right)>0$ or $q_{2}\left(\tau^{+}\right)<0$.

Analogously, either $\lim _{t \rightarrow-\infty} q(t)=(-1,0)$ or $q(t)$ had to enter $\mathcal{C}$ at some negative entrance time $\tau^{-}\left(q(0), q^{\prime}(0)\right)$ contimuous in $\left(q(0), q^{\prime}(0)\right)$. Also, in the second case, $q^{\prime}\left(\tau^{-}\right) \neq 0$ belongs to $\partial K_{0} \cap \partial K_{1}$ or $\partial K_{0} \cap \partial K_{3}$ if, respectively, $q_{2}\left(\tau^{-}\right)<0$ or $q_{2}\left(\tau^{-}\right)>0$.

In the following lemma, $\phi(t, q, v) \in \mathbb{R}^{2}$ denotes the solution of (EFK') satisfying $\phi(0, q, v)=q$ and $\phi^{\prime}(0, q, v)=v$ (derivative with respect to time). Note that the maximal time interval where $\phi(t, v, q)$ is defined is not necessarily all $\mathbb{R}$. The aim of this lemma is to describe qualitatively the part of the local unstable manifold $W_{\text {loc }}^{\mathrm{u}}(-1)$ that is 'over' $\overline{\mathcal{C}}$ in terms of the variables $(q, v)$ and the monotonicity property of paths. More precisely, for $\epsilon>0$ small enough, we set

$$
\mathcal{A}:=\left\{(q, v) \in W_{\mathrm{loc}}^{\mathrm{u}}(-1): q \in \overline{\mathcal{C}}, q_{1}=-1+\epsilon\right\} .
$$

Intuitively, $\mathcal{A}$ is the part of $W_{\text {loc }}^{\mathrm{u}}(-1)$ from which we shall shoot at the local stable manifold $W_{\text {loc }}^{\mathrm{s}}(1)$.

LEMMA 5.1. Let $\gamma \in\left(0, \frac{1}{8}\right]$ be fixed. For small $\epsilon>0$ and all $(q, v) \in \mathcal{A}$, we have $v \in \bar{K}_{0} \backslash\{0\}$ and $\phi(t, q, v) \in \mathcal{C}$ for all negative $t$. Moreover, to each $q \in \overline{\mathcal{C}}$ with 
$q_{1}=-1+\epsilon$ corresponds a unique $v \in \bar{K}_{0}$ such that $(q, v) \in W_{\mathrm{loc}}^{\mathrm{u}}(-1)$. If, in addition, $q \in \partial \mathcal{C}$, then $v \in \partial K_{0} \cap \partial K_{1}$ when $q_{2}>0$ and $v \in \partial K_{0} \cap \partial K_{3}$ when $q_{2}<0$.

Proof. This lemma is a consequence of the facts that $W_{\text {loc }}^{\mathrm{u}}(-1)$ is tangent to the unstable manifold of the linearized problem around $u=-1$, for which explicit computations can be made, and the monotonicity property of paths. For example, let us prove that $\tau^{-}(q, v)=-\infty$. Ad absurdum, suppose $\tau^{-}(q, v)$ finite and $\phi_{2}\left(\tau^{-}, q, v\right)>0$, say. Then $\phi^{\prime}\left(\tau^{-}, q, v\right) \in \partial K_{0} \cap \partial K_{3}$ and $\phi^{\prime}(t, q, v) \in K_{3}$ for $t<\tau^{-}$ near $\tau^{-}$. We can suppose that $\tilde{q}_{1}<0$ for all $(\tilde{q}, \tilde{v}) \in W_{\mathrm{loc}}^{\mathrm{u}}(-1)$ and, in particular, that $\phi_{1}(t, q, v)<0$ for all $t \leqslant 0$. A picture shows that $\phi(t, q, v) \notin V_{0}$ and $\phi^{\prime}(t, q, v) \in K_{3}$ for all $t<\tau^{-}$. Hence $\lim _{t \rightarrow-\infty}|\phi(t, q, v)|=\infty$. Contradiction.

We now introduce a homotopy parameter $-1<x<1$ as follows. Let $q(t)$ be a solution of $\left(\mathrm{EFK}^{\prime}\right)$ with zero Hamiltonian and such that $q(0) \in \overline{\mathcal{C}}$ and $q^{\prime}(0) \in \bar{K}_{0}$. For $-1<q_{1}(0) \leqslant x<1$, the path $q(t)$ has to leave the region $\left\{w \in \overline{\mathcal{C}}: w_{1} \leqslant x\right\}$ at some finite exit time $\tau_{x}^{+}\left(q(0), q^{\prime}(0)\right) \geqslant 0$ that is continuous in $\left(q(0), q^{\prime}(0)\right)$. The corresponding leaving point in $\mathbb{R}^{4}$ is given by

$$
\Gamma_{x}(z):=\left(\phi, \phi^{\prime}\right)\left(\tau_{x}^{+}(z), z\right),
$$

where $z=\left(q(0), q^{\prime}(0)\right)$. The value 0 for the homotopy parameter $x$ will play a distinguished role, as well as the following subset of $W^{\mathrm{u}}(-1)$ :

$$
M:=\Gamma_{0}(\mathcal{A}) \subset W^{\mathrm{u}}(-1) .
$$

The image of $M$ under the symmetry $S(q, v)=(-q, v) \in \mathbb{R}^{4}$ clearly satisfies $S M \subset W^{\mathrm{s}}(1)$.

ThEOREM 5.2. Let $\gamma \in\left(0, \frac{1}{8}\right]$ be fixed. The sets $M$ and $S M$ have a compact intersection included in

$$
Y:=\left\{(q, v) \in H_{0}: q \in \mathcal{C}, v \in K_{0}, q_{1}=0\right\} .
$$

Restricting $M$ and $S M$ to an appropriate closed subset of $Y$, the restrictions are compact smooth orientable manifolds with boundary that are embedded in $Y$ and admissible for intersection theory. Their orientations are given by the images by the flow of orientations on $\mathcal{A}$ and $S \mathcal{A}$. Their intersection number, denoted by $I(M, S M)$, is equal to \pm 1 .

REMARK 5.3. The symmetry $S$ has only been introduced for the sake of simplicity.

Proof. Let $\tilde{S}: \mathbb{R}^{4} \rightarrow \mathbb{R}^{4}$ be the symmetry $\tilde{S}\left(q_{1}, q_{2}, v_{1}, v_{2}\right)=\left(q_{1},-q_{2}, v_{1}, v_{2}\right)$. Note that $S M$ and $\tilde{S} M$ coincide on $Y$. Set

$$
F_{x}=\left\{q \in \partial \mathcal{C}:-1+\epsilon \leqslant q_{1} \leqslant x\right\} \cup\left\{q \in \mathcal{C}: q_{1}=x\right\} .
$$

$F_{x}$ is homeomorphic to the segment $[-1,1]$. Let $\alpha_{x}: F_{x} \rightarrow[-1,1]$ be a homeomorphism. We may suppose $\alpha_{x}(q)$ to be continuous both in $x$ and $q$ and differentiable if $q \in \mathcal{C}$. Finally, we define

$$
\beta_{x}:\left\{\begin{array}{l}
\left(F_{x} \times \bar{K}_{0}\right) \cap H_{0} \rightarrow[-1,1] \times \mathbb{R} \\
\beta_{x}(q, v)=\left(\alpha_{x}(q), v_{2}\right)
\end{array}\right.
$$


which is also a homeomorphism and is differentiable in $q$ and $x$ if $q \in \mathcal{C}$. In what follows, the domains of the maps $\beta_{x} \circ \Gamma_{x}$ and $\beta_{x} \circ \tilde{S} \circ \Gamma_{x}$ are taken to be $\mathcal{A}$, and their ranges are included in $\mathbb{R}^{2}$. We obtain

$$
\begin{aligned}
I(M, S M) & =I\left(\Gamma_{0}(\mathcal{A}), S \Gamma_{0}(\mathcal{A})\right) \\
& =I\left(\Gamma_{0}(\mathcal{A}), \tilde{S} \Gamma_{0}(\mathcal{A})\right) \\
& = \pm I\left(\beta_{0} \circ \Gamma_{0}, \beta_{0} \circ \tilde{S} \circ \Gamma_{0}\right) \\
& = \pm I\left(\beta_{x} \circ \Gamma_{x}, \beta_{x} \circ \tilde{S} \circ \Gamma_{x}\right)
\end{aligned}
$$

for all $x \in[-1+\epsilon, 0]$, because the parameter $x$ describes a homotopy whose admissibility results from $\Gamma_{x}(\partial \mathcal{A})=\partial \mathcal{A},\left(\tilde{S} \circ \Gamma_{x}\right)(\partial \mathcal{A})=\tilde{S}(\partial \mathcal{A})$ and $\partial A \cap \tilde{S}(\partial \mathcal{A})=\emptyset$. Hence

$$
\begin{aligned}
I(M, S M) & = \pm I\left(\beta_{-1+\epsilon}, \beta_{-1+\epsilon} \circ \tilde{S}\right) \\
& = \pm I\left(\beta_{-1+\epsilon}(\mathcal{A}), \beta_{-1+\epsilon}(\tilde{S} \mathcal{A})\right)
\end{aligned}
$$

(which is equal to $I(\mathcal{A}, \widetilde{S} \mathcal{A})$ ). There is a smooth transformation of $\mathbb{R}^{2}$ which changes $\beta_{-1+\epsilon}(\mathcal{A})$ and $\beta_{-1+\epsilon}(\tilde{S} \mathcal{A})$ into $\{(a, a):|a| \leqslant 1\}$ and $\{(-a, a):|a| \leqslant 1\}$, without the boundaries being involved in the intersection during the process. We deduce $I(M, S M)= \pm 1$. Incidentally, we also obtain the relation $I(M, S M)=I(\mathcal{A}, \tilde{S} \mathcal{A})$, the ambient manifold for the left-hand member being $Y$ and the one for the righthand member $\left\{(q, v) \in H_{0}: q_{1}=-1+\epsilon\right\}$.

COROLlary 5.4. There exists $\epsilon>0$ such that for all $\frac{1}{8}<\gamma<\frac{1}{8}+\epsilon$, (EFK) is chaotic and admits infinitely many kinks connecting -1 to 1 .

Proof. The sets $M$ and $S M$ still intersect each other in $Y$ for $\gamma>\frac{1}{8}$ near $\frac{1}{8}$ thanks to the topological transversality of the intersection for $\gamma=\frac{1}{8}$. Thus (EFK) admits a kink from $u \equiv-1$ to $u \equiv 1$ and the reversibility of (EFK) gives another one from $u \equiv 1$ to $u \equiv-1$. Using the fact that $u \equiv-1$ and $u \equiv 1$ are saddle-foci for $\gamma>\frac{1}{8}$, and arguing as in $\S 3$, we can prove that (EFK) is chaotic.

This last corollary is of the same flavour of a similar result known for the waterwave equation (1.1) (see [9]) and is a contribution to the understanding of the chaotic dynamics of (EFK) (see [22,23,31]).

\section{The case $\gamma>\frac{1}{8}$}

Throughout this section, we assume that $\gamma>\frac{1}{8}$. An infinite family of kinks is found in [30] by a shooting method, starting from the invariant set $S_{0}:=\left\{(q, v) \in \mathbb{R}^{4}\right.$ : $q=0\}$ of the symmetry $S(q, v)=(-q, v)$. There are two difficulties when trying to prove some kind of transversality. First, the stable and unstable manifolds do not play an explicit role in the shooting method and, second, the kinks are no more monotonic. To cope with these difficulties, we shall use the analyticity of (EFK) and rely on the Hopf degree theorem. More specifically, we shall need the following result.

Proposition 6.1. Let $M_{1}$ and $M_{2}$ be two compact pieces of manifolds embedded in an orientable manifold $Y$ such that $\operatorname{dim} M_{1}=\operatorname{dim} M_{2}=\operatorname{dim} \frac{1}{2} Y$ and 
$M_{1} \cap M_{2}=\{y\}$. We denote the inclusion mapping of $M_{1}$ in $Y$ by $j \in C^{1}\left(M_{1}, Y\right)$. If $M_{1}$ and $M_{2}$ are admissible for intersection theory and $I\left(M_{1}, M_{2}\right)=0$, then there exists an embedding $f \in C^{1}\left(M_{1}, Y\right)$ such that $f^{-1}\left(M_{2}\right)=\emptyset ; f$ may be chosen to be as near to $j$ in $C\left(M_{1}, Y\right)$ as necessary and to be equal to $j$ outside an open set of $M_{1}$ that is as small as necessary.

Proof. Let $\operatorname{dim} Y=2 m$. Restricting ourselves to smaller compact pieces of $M_{1}$ and $M_{2}$ and using local coordinates $\left(y_{1}, y_{2}\right) \in \mathbb{R}^{2 m}$ with $y_{i} \in \mathbb{R}^{m}$ for $i=1,2$, we may suppose without loss of generality that $Y=\mathbb{R}^{2 m}, y=0, M_{1}=\left\{\left(y_{1}, y_{2}\right): y_{1} \in\right.$ $\left.\bar{B}_{\epsilon}, y_{2}=0\right\}$ and $M_{2}=\left\{\left(y_{1}, g\left(y_{1}\right)\right): y_{1} \in \bar{B}_{\epsilon}\right\}$ for some small $\epsilon>0$ and some map $g \in C^{1}\left(\bar{B}_{\epsilon}, \mathbb{R}^{m}\right)$ such that $g(0)=0$. We use the notation $\vec{B}_{\epsilon}:=\left\{y \in \mathbb{R}^{m}:\|y\| \leqslant \epsilon\right\}$.

The definition of the topological degree of a map gives

$$
\operatorname{deg}\left(g, 0, \bar{B}_{\epsilon}\right)= \pm I\left(M_{1}, M_{2}\right)=0 .
$$

By the Hopf degree theorem $[16,18]$, there exists a map $\tilde{g} \in C^{1}\left(\bar{B}_{\epsilon}, \mathbb{R}^{m}\right)$ which never takes the value 0 , which is as close as necessary to $g$ in $C\left(\bar{B}_{\epsilon}, \mathbb{R}^{m}\right)$ and which is equal to $g$ outside an open set that is as small as necessary.

Define $f: M_{1} \rightarrow Y$ by $f\left(y_{1}, 0\right)=\left(y_{1}, g\left(y_{1}\right)-\tilde{g}\left(y_{1}\right)\right)$.

Following [30], for $\alpha \neq 0$, we denote by $u(., \alpha)$ the solution of (EFK) such that $u(0, \alpha)=u^{\prime \prime}(0, \alpha)=0, u^{\prime}(0, \alpha)=\alpha$ and $u^{\prime \prime \prime}(0, \alpha)=(2 \alpha \gamma)^{-1}\left(\alpha^{2}-\frac{1}{2}\right)$. Clearly, $\left(u, u^{\prime \prime}, u^{\prime}, u^{\prime \prime \prime}\right)(0, \alpha)$ is in $S_{0} \cap H_{0}$. We use the notation $z(., \alpha)=\left(u, u^{\prime \prime}, u^{\prime}, u^{\prime \prime \prime}\right)(., \alpha)$. Inspecting [30], we obtain the following result, which is based on a shooting method. Let $W_{T}^{\mathrm{s}}(1)$ be the set of points in $\mathbb{R}^{4}$ that are sent by the flow in $W_{\text {loc }}^{\mathrm{s}}(1)$ after a period of time $T$, and let $W_{T}^{\mathrm{u}}(-1)=S W_{T}^{\mathrm{s}}(1)$ be the set of points in be the set of points in $\mathbb{R}^{4}$ that are sent by the backward flow in $W_{\text {loc }}^{\mathrm{u}}(-1)$ after a period of time $T$.

THEOREM 6.2. There exist $T>0$ and a sequence of compact intervals $\left(\left[a_{i}, b_{i}\right]\right.$, $i \in \mathbb{N})$ such that $0<a_{i}<a_{i+1}<b_{i+1}<b_{i}(i \in \mathbb{N})$ and which satisfy the following properties.

(i) Kink: for all $\alpha \in \cap_{i \in \mathbb{N}}\left[a_{i}, b_{i}\right]$, the solution $z(0, \alpha)$ is in $W_{T}^{\mathrm{s}}(1)$.

(ii) Periodic solutions: in each interval $\left[a_{i}, b_{i}\right]$, there exists $\alpha$ such that $z(., \alpha)$ is a periodic orbit.

(iii) Persistence under perturbation: if $\gamma \in C\left(\left[a_{i}, b_{i}\right], \mathbb{R}^{4}\right)$ is such that $\gamma\left(a_{i}\right)=$ $z\left(0, a_{i}\right), \gamma\left(b_{i}\right)=z\left(0, b_{i}\right)$ and $\gamma$ is sufficiently close to $z(0,$.$) in C\left(\left[a_{i}, b_{i}\right], \mathbb{R}^{4}\right)$, then there exists $\alpha \in\left[a_{i}, b_{i}\right]$ such that $\gamma(\alpha) \in W_{T}^{\mathrm{s}}(1)$.

We now prove the following theorem.

THEOREM 6.3. We have $\lim a_{i}=\lim b_{i}:=\alpha_{\infty}$ and $z\left(0, \alpha_{\infty}\right)$ is a topological transverse intersection point of $W_{T}^{\mathrm{s}}(1)$ and $S_{0}$ in

$$
Y:=\left\{(q, v) \in H_{0}: q \in \mathcal{C}, v \in K_{0}, q_{1}=0\right\} .
$$

Proof. Ad absurdum, suppose that $\lim a_{i} \neq \lim b_{i}$. Denote the limits by, respectively, $\alpha_{\infty}$ and $\beta_{\infty}$. By the first property of the preceding theorem, $S_{0} \cap W_{T}^{\text {s }}(1) \cap Y$ contains 
the 1-manifold $\left\{z(0, \alpha): \alpha_{\infty} \leqslant \alpha \leqslant \beta_{\infty}\right\}$. As $S_{0} \cap Y$ and $W_{T}^{\mathbf{s}}(1) \cap Y$ are analytical 1-manifolds embedded in the analytical 2-manifold $Y$, there exists an interval $(a, b)$ containing $\left[\alpha_{\infty}, \beta_{\infty}\right]$, and such that $z(0, \alpha) \in S_{0} \cap W_{T}^{\mathrm{s}}(1) \cap Y$ for all $\alpha \in(a, b)$. As $\left[a_{i}, b_{i}\right] \subset(a, b)$ for large $i \in \mathbb{N}$, this contradicts the second property of the preceding theorem. Therefore, $\alpha_{\infty}=\beta_{\infty}$ and, again by analyticity, $z\left(0, \alpha_{\infty}\right)$ is an isolated point of $S_{0} \cap W_{T}^{\mathrm{s}}(1) \cap Y$.

Suppose now that the intersection is not topologically transverse and let us find a contradiction. By proposition 6.1 , given some small neighbourhood of $z\left(0, \alpha_{\infty}\right)$ in $Y$, it is possible to perturb slightly $S_{0} \cap Y$ inside this neighbourhood in such a way that the perturbed $C^{1}$ manifold of dimension one does not intersect $W_{T}^{\mathrm{s}}(1) \cap Y$ (in this neighbourhood). Hence if we start shooting from the perturbed manifold, orbits do not reach $W_{\text {loc }}^{\mathrm{s}}(1)$ by time $T$. This contradicts the third property of the preceding theorem:

The next task is to deduce the topological transversality of $W^{\mathrm{u}}(-1)$ and $W^{\mathrm{s}}(1)$ in $Y$. We shall shoot directly from $W_{\text {loc }}^{\mathrm{u}}(-1)$ and use the following proposition that is proved in the same way as theorems 6.2 and 6.3 .

Proposition 6.4. For $\alpha \in \mathbb{R}$, let $t \rightarrow \tilde{u}(t, \alpha)$ be a solution of (EFK) such that $\tilde{z}(t, \alpha):=\left(\tilde{u}, \tilde{u}^{\prime \prime}, \tilde{u}^{\prime}, \tilde{u}^{\prime \prime \prime}\right)(t, \alpha)$ satisfy $\tilde{z}(0, \alpha) \in W_{\text {loc }}^{\mathrm{u}}(-1) \backslash\{(-1,0,0,0)\}$. We suppose $\tilde{z}(0, \alpha)$ to be real analytic in $\alpha$ and we assume that there exists $T>0$ and a sequence of compact intervals $\left(\left[a_{i}, b_{i}\right], i \in \mathbb{N}\right)$ such that $a_{i}<a_{i+1}<b_{i+1}<b_{i}(i \in \mathbb{N})$ and which satisfy the following properties $\left(T, a_{i}\right.$ and $b_{i}$ may be different from those of theorem 6.2).

(i) Kink: for all $\alpha \in \cap_{i \in \mathbb{N}}\left[a_{i}, b_{i}\right]$, the solution $\tilde{z}(0, \alpha)$ is in $W_{T}^{\mathrm{s}}(1)$.

(ii) In each interval $\left[a_{i}, b_{i}\right]$, there exists $\alpha$ such that $\tilde{z}(., \alpha) \notin W_{T}^{\mathrm{s}}(1)$.

(iii) Persistence under perturbation: if $\gamma \in C\left(\left[a_{i}, b_{i}\right], \mathbb{R}^{4}\right)$ is such that $\gamma\left(a_{i}\right)=$ $\tilde{z}\left(0, a_{i}\right), \gamma\left(b_{i}\right)=\tilde{z}\left(0, b_{i}\right)$ and $\gamma$ is sufficiently close to $\tilde{z}(0,$.$) in C\left(\left[a_{i}, b_{i}\right], \mathbb{R}^{4}\right)$, then there is $\alpha \in\left[a_{i}, b_{i}\right]$ such that $\gamma(\alpha) \in W_{T}^{\mathrm{s}}(1)$.

Then there exists a kink that is the topological transverse intersection of $W_{\mathrm{loc}}^{\mathrm{u}}(-1)$ and $W_{T}^{\mathrm{s}}(1)$ restricted to an appropriate Poincaré section.

We shall also need the following lemma.

LEMMA 6.5. Let $M_{1}$ and $M_{2}$ be two compact pieces of one-dimensional manifolds that are embedded in $\mathbb{R}^{2}$, admissible for intersection theory and such that $M_{1} \cap M_{2}=\{0\}$. Let $\bar{S}: \mathbb{R}^{2} \rightarrow \mathbb{R}^{2}$ be the linear symmetry $\bar{S}\left(y_{1}, y_{2}\right)=\left(y_{1},-y_{2}\right)$, $y_{1}, y_{2} \in \mathbb{R}$, and let $\bar{S}_{0}=\left\{\left(y_{1}, y_{2}\right) \in \mathbb{R}^{2}: y_{2}=0\right\}$ be its invariant set. If $M_{2}=\bar{S} M_{1}$ and $I\left(M_{1}, \bar{S}_{0}\right) \neq 0$, then $I\left(M_{1}, M_{2}\right) \neq 0$.

This lemma, which is inspired from $\S 4.2$ of [6], implies the following alternative: either $z\left(0, \alpha_{\infty}\right)$ (given by theorem 6.3) is a topological transverse intersection point of $W_{T}^{\mathrm{u}}(-1) \cap Y$ and $W_{T}^{\mathrm{s}}(1) \cap Y$, or these two manifolds coincide locally near $z\left(0, \alpha_{\infty}\right)$.

Proof of the lemma. Let us first suppose $M_{1}$ tangent to $\bar{S}_{0}$. We can find $h \in$ $C^{1}([-\epsilon, \epsilon], \mathbb{R})$ such that $M_{1}=\left\{\left(y_{1}, h\left(y_{1}\right)\right):\left|y_{1}\right| \leqslant \epsilon\right\}$ (restricting $M_{1}$ to a smaller 
piece if necessary). We obtain $M_{2}=\left\{\left(y_{1},-h\left(y_{1}\right)\right):\left|y_{1}\right| \leqslant \epsilon\right\}$ and

$$
I\left(M_{1}, M_{2}\right)= \pm \operatorname{deg}(2 h,[-\epsilon, \epsilon], 0)= \pm \operatorname{deg}(h,[-\epsilon, \epsilon], 0)= \pm I\left(M_{1}, \bar{S}_{0}\right) \neq 0 .
$$

Second, suppose that $M_{1}$ is not tangent to $\bar{S}_{0}$. Then we can find $h \in C^{1}([-\epsilon, \epsilon], \mathbb{R})$ such that $M_{1}=\left\{\left(h\left(y_{2}\right), y_{2}\right):\left|y_{2}\right| \leqslant \epsilon\right\}$ (restricting $M_{1}$ to a smaller piece if necessary). We obtain $M_{2}=\left\{\left(h\left(-y_{2}\right), y_{2}\right):\left|y_{2}\right| \leqslant \epsilon\right\}$ and

$$
I\left(M_{1}, M_{2}\right)=\operatorname{deg}(h(x)-h(-x),[-\epsilon, \epsilon], 0)= \pm 1
$$

(the map is odd).

THEOREM 6.6. There exists a topologically transverse intersection point of

$$
W^{\mathrm{u}}(-1) \cap Y \quad \text { and } \quad W^{\mathrm{s}}(1) \cap Y
$$

in

$$
Y=\left\{(q, v) \in H_{0}: q \in \mathcal{C}, v \in K_{0}, q_{1}=0\right\} .
$$

Sketch of proof. The idea is to start shooting from $W_{\mathrm{loc}}^{\mathrm{u}}(-1)$, using the existence of $z\left(., \alpha_{\infty}\right)$. We thus manage to obtain some topologically transverse intersection point of $W^{\mathrm{u}}(-1) \cap Y$ and $W^{\mathrm{s}}(1) \cap Y$ (via proposition 6.4) or a sequence $\left(z_{n} ; n \in \mathbb{N}\right) \subset W_{T}^{\mathrm{u}}(-1) \cap W_{\tau_{n}}^{\mathrm{s}}(-1) \cap Y$ whose limit belongs to the same component of $W_{T}^{\mathrm{u}}(-1) \cap W_{T}^{\mathrm{s}}(1) \cap Y$ as $z\left(0, \alpha_{\infty}\right)$ (increasing the value of $T$ if necessary).

Let us focus on the second case. First, note that every point $z_{n}$ belongs to a homoclinic orbit to -1 that spends a period of time near 1 that tends to infinity with $n$. By the analyticity of $W_{T}^{\mathrm{u}}(-1) \cap Y, W_{T}^{\mathrm{s}}(1) \cap Y$ and $Y, \lim z_{n}$ is an isolated intersection point of $W_{T}^{\mathrm{u}}(-1) \cap Y$ and $W_{T}^{\mathrm{s}}(1) \cap Y$, otherwise $W_{T}^{\mathrm{u}}(-1) \cap W_{T}^{\mathrm{s}}(1) \cap Y$ would include a 1-manifold that contains $\lim z_{n}$ and $z_{n}$ for large $n$, in contradiction with $z_{n} \in W^{\mathrm{s}}(-1)$. Hence $\lim z_{n}=z\left(0, \alpha_{\infty}\right)$ and we conclude by applying lemma 6.5 (via some local chart) to $W_{T}^{\mathrm{s}}(1)=S W_{T}^{\mathrm{u}}(1), W_{T}^{\mathrm{u}}(1)$ and $S_{0}$ restricted to the twodimensional manifold $Y$.

This gives a new proof that the extended Fisher-Kolmogorov equation is chaotic for all values of $\gamma>\frac{1}{8}$ (see $\left.[22,23,31]\right)$.

\section{Travelling kinks}

A more general form of the EFK equation reads as follows (see, for example, [29]):

$$
\frac{\partial}{\partial t} u(t, x)=-\gamma \frac{\partial^{4} u}{\partial x^{4}}+\frac{\partial^{2} u}{\partial x^{2}}+(u-a)\left(1-u^{2}\right), \quad-1<a<1, \quad \gamma>0 .
$$

Stationary travelling waves propagating at speed $c \in \mathbb{R}$ are described by the equation

$$
c u^{\prime}(t)-\gamma u^{(i v)}(t)+u^{\prime \prime}(t)+(u-a)\left(1-u^{2}\right)=0, \quad-1<a<1, \quad \gamma>0
$$

(we denote the independent variable by $t$ instead of $x$ ). When $a=c=0$, equation (7.1) is simply the EFK equation introduced in $\S 4$. Our aim is to show that topologically transverse kinks when $a=c=0$ persist for $c \neq 0$ small. 
THEOREM 7.1. Let $\gamma>0, a=c=0$,

$$
Y=\left\{(q, v) \in H_{0}: q \in \mathcal{C}, v \in K_{0}, q_{1}=0\right\},
$$

and $M_{\mathrm{s}} \subset W_{T}^{\mathrm{s}}(1) \cap Y$ and $M_{\mathrm{u}} \subset W_{T}^{\mathrm{u}}(-1) \cap Y$ be two compact pieces of manifolds that are admissible for intersection theory and such that $I\left(M_{\mathrm{u}}, M_{\mathrm{s}}\right) \neq 0$ in the manifold $Y$ (for some $T>0$ ). Then there exist $a_{0} \in(0,1)$ and $c_{0}>0$ such that, for all $a \in\left(-a_{0}, a_{0}\right)$, it is possible to find $c \in\left(-c_{0}, c_{0}\right)$ and $u \in C^{\infty}(\mathbb{R})$ such that (7.1) holds and $\lim _{t \rightarrow \pm \infty} u(t)= \pm 1$. Moreover, $c \neq 0$ if $a \neq 0$.

Proof. Note that

$$
H\left(u, u^{\prime}, u^{\prime \prime}, u^{\prime \prime \prime}\right):=2 \gamma u^{\prime} u^{\prime \prime \prime}-\gamma\left(u^{\prime \prime}\right)^{2}-\left(u^{\prime}\right)^{2}+\frac{1}{2}\left(1-u^{2}\right)^{2}+2 a\left(u-\frac{1}{3} u^{3}\right)
$$

is an invariant for $c=0$ and a Lyapunov function for $c \neq 0$; indeed,

$$
\begin{aligned}
\frac{\mathrm{d}}{\mathrm{d} t} H\left(u, u^{\prime}, u^{\prime \prime}, u^{\prime \prime \prime}\right) & =2\left\{\gamma u^{(i v)}-u^{\prime \prime}-(u-a)\left(1-u^{2}\right)-c u^{\prime}\right\} u^{\prime}+2 c u^{\prime}(t)^{2} \\
& =2 c u^{\prime}(t)^{2}
\end{aligned}
$$

has the same sign as $c$ for all $t$ and any solution $u \in C^{4}(\mathbb{R})$. Let $Z$ be the threedimensional manifold $Z=\left\{(q, v) \in \mathbb{R}^{4}: q \in \mathcal{C}, v \in K_{0}, q_{1}=0\right\}, W^{\mathrm{s}}(a, c)$ be a compact piece of the manifold $W_{T}^{\mathrm{s}}(1)$ for equation $(7.1)$ and $W^{\mathrm{u}}(a, c)$ be a compact piece of the manifold $W_{T}^{\mathrm{u}}(-1)$ for equation (7.1) with $a$ and $c$ arbitrary (but small). We assume that $W^{\mathrm{u}}(0,0) \cap Z=M_{\mathrm{u}}$ and $W^{\mathrm{s}}(0,0) \cap Z=M_{\mathrm{s}}$. We also suppose that the sets $\cup_{|c| \leqslant c_{0}} W^{\mathrm{u} / \mathrm{s}}(a, c) \times\{c\}$ are two compact pieces of manifolds for $|a|<a_{0}$ and $c_{0}, a_{0}>0$ small enough.

As a consequence, for $|a|<a_{0}$, the sets

$$
N_{\mathrm{u} / \mathrm{s}}(a)=\cup_{|c| \leqslant c_{0}}\left(W^{\mathbf{u} / \mathrm{s}}(a, c) \cap Z\right) \times\{c\} \subset Z \times \mathbb{R}
$$

are two compact pieces of two-dimensional manifolds. Let us show that, for $a=0$, they are admissible for intersection theory and $I\left(N_{\mathrm{u}}(0), N_{\mathrm{s}}(0)\right) \neq 0$ in $Z \times \mathbb{R}$. We can assume that

$$
x \in \operatorname{int}\left(W^{\mathrm{u} / \mathrm{s}}(a, c) \cap Z\right) \Rightarrow(x, c) \in \operatorname{int} N^{\mathrm{u} / \mathrm{s}}(a)
$$

for all $|c|<c_{0}$ and $|a|<a_{0}$.

By equation (7.2), for $c>0$, the value of $H$ is positive on $W^{\mathrm{u}}(0, c) \cap Z$ and negative on $W^{\mathrm{s}}(0, c) \cap Z$. For $c<0$, the value of $H$ is negative on $W^{\mathrm{u}}(0, c) \cap Z$ and positive on $W^{\mathrm{s}}(0, c) \cap Z$. In short, the value of $c H$ is positive on $W^{\mathrm{u}}(0, c) \cap Z$ and negative on $W^{\mathrm{s}}(0, c) \cap Z$ if $c \neq 0$. As a consequence, $N_{\mathrm{u}}(0)$ and $N_{\mathrm{s}}(0)$ intersect each other in $Z \times\{0\}$. Therefore, the admissibility of $N_{\mathrm{u}}(0)$ and $N_{\mathrm{s}}(0)$ results from the one of $M_{\mathrm{u}}$ and $M_{\mathrm{s}}$.

The manifold $Z$ admits a global chart $\phi: Z \rightarrow \mathbb{R}^{3}$ given by

$$
\phi(q, v, c)=\left(q_{2}, v_{2}, H(q, v)\right)
$$

We now embed each of $(\phi \times I)\left(N_{\mathrm{u}}(0)\right)$ and $(\phi \times I)\left(N_{\mathrm{s}}(0)\right)$ in a continuous family of 2-manifolds in $\mathbb{R}^{4}$ in such a way to obtain an admissible homotopy, which we 
parametrize by $\theta \in[0,1]$. We set

$$
\begin{aligned}
& \tilde{N}_{\mathrm{u}}(\theta)=\left\{\left(q_{2}, v_{2}, h+\theta c, c\right):\left(q_{2}, v_{2}, h\right) \in \phi\left(W^{\mathrm{u}}(0, c-\theta c) \cap Z\right),|c| \leqslant c_{0}\right\}, \\
& \tilde{N}_{\mathrm{s}}(\theta)=\left\{\left(q_{2}, v_{2}, h-\theta c, c\right):\left(q_{2}, v_{2}, h\right) \in \phi\left(W^{\mathrm{s}}(0, c-\theta c) \cap Z\right),|c| \leqslant c_{0}\right\} .
\end{aligned}
$$

Clearly, $\tilde{N}_{\mathrm{u} / \mathrm{s}}(0)=(\phi \times I)\left(N_{\mathrm{u} / \mathrm{s}}(0)\right)$ and

$$
\begin{aligned}
& \tilde{N}_{\mathrm{u}}(1)=\left\{\left(q_{2}, v_{2}, c, c\right):\left(q_{2}, v_{2}, 0\right) \in \phi\left(W^{\mathrm{u}}(0,0) \cap Z\right),|c| \leqslant c_{0}\right\}, \\
& \tilde{N}_{\mathrm{s}}(1)=\left\{\left(q_{2}, v_{2},-c, c\right):\left(q_{2}, v_{2}, 0\right) \in \phi\left(W^{\mathrm{s}}(0,0) \cap Z\right),|c| \leqslant c_{0}\right\} .
\end{aligned}
$$

We now check the admissibility of the homotopy. Let

$$
\left(q_{2}^{\mathrm{u}}, v_{2}^{\mathrm{u}}, h^{\mathrm{u}}+\theta c^{\mathrm{u}}, c^{\mathrm{u}}\right) \in \tilde{N}_{\mathrm{u}}(\theta)
$$

and

$$
\left(q_{2}^{\mathrm{s}}, v_{2}^{\mathrm{s}}, h^{\mathrm{s}}-\theta c^{\mathrm{s}}, c^{\mathrm{s}}\right) \in \tilde{N}_{\mathrm{s}}(\theta)
$$

be equal. We get $c^{\mathrm{u}}=c^{\mathrm{s}}:=c$ and $c\left(h^{\mathrm{s}}-h^{\mathrm{u}}\right)=2 c^{2} \theta \geqslant 0$. As $c\left(h^{\mathrm{s}}-h^{\mathrm{u}}\right)<0$ if $c \neq 0$, we deduce that $c=0, h^{\mathrm{u}}=h^{\mathrm{s}}=0$,

$$
\left(q_{2}^{\mathrm{u}}, v_{2}^{\mathrm{u}}, 0\right) \in \phi\left(W^{\mathrm{u}}(0,0) \cap Z\right)
$$

and

$$
\left(q_{2}^{\mathrm{s}}, v_{2}^{\mathrm{s}}, 0\right) \in \phi\left(W^{\mathrm{s}}(0,0) \cap Z\right) .
$$

Thus the admissibility of $\tilde{N}_{\mathrm{u}}(\theta)$ and $\tilde{N}_{\mathrm{s}}(\theta)$ follows from the one of $M_{\mathrm{u}}$ and $M_{\mathrm{s}}$. By homotopy,

$$
\begin{aligned}
\left.I\left(N_{\mathrm{u}}(a), N_{\mathrm{s}}(a)\right)\right|_{a=0} & = \pm\left. I\left(\tilde{N}_{\mathrm{u}}(\theta), \tilde{N}_{\mathrm{s}}(\theta)\right)\right|_{\theta=0} \\
& = \pm\left. I\left(\tilde{N}_{\mathrm{u}}(\theta), \tilde{N}_{\mathrm{s}}(\theta)\right)\right|_{\theta=1} \\
& = \pm I\left(M_{\mathrm{u}} \times\left\{(c, c):|c| \leqslant c_{0}\right\}, M_{\mathrm{s}} \times\left\{(-c, c):|c| \leqslant c_{0}\right\}\right) \\
& = \pm I\left(M_{\mathrm{u}}, M_{\mathrm{s}}\right) \neq 0 .
\end{aligned}
$$

This persists under small perturbations, and hence there exists $a_{0} \in(0,1)$ such that $I\left(N_{\mathrm{u}}(a), N_{\mathrm{s}}(a)\right) \neq 0$ in $Z \times \mathbb{R}$ for all $|a|<a_{0}$. As a consequence, for all $|a|<a_{0}$, there exists $c \in\left(-c_{0}, c_{0}\right)$ such that the manifolds $W^{\mathrm{u}}(a, c)$ and $W^{\mathrm{s}}(a, c)$ intersect each other in $Z$.

If $a \neq 0$ and $c=0$, the invariant $H$ takes two different values at $u \equiv 1$ and $u \equiv-1$ and, therefore, no kink can connect these two stationary solutions.

\section{Acknowledgments}

The author thanks Patrick Bernard and Eric Séré (Paris-Dauphine), who described to him the work of Easton [15].

This paper was mainly written when the author visited the Department of Mathematics of the University of Leiden (September 1996), whose hospitality is gratefully acknowledged. There, Professor L. A. Peletier asked whether topological transversality may be used in proving the existence of slow travelling kinks for the extended Fisher-Kolmogorov equation (see $\S 7$ ). 


\section{Appendix A. Topological transversality}

We call $X$ a compact piece of manifold if $X$ is a non-empty compact subset of some boundaryless and orientable $C^{1}$ manifold $\tilde{X}$ of finite dimension such that $X=\operatorname{int} X$ in $\tilde{X}$. We endow $X$ with the induced topology. For a $C^{1}$ manifold $Y$, we write $f \in C^{1}(X, Y)$ if $f$ can be extended to a map $\tilde{f} \in C^{1}(\tilde{X}, Y)$, and we say that $f$ is a $C^{1}$ embedding if, moreover, $\tilde{f}$ is a $C^{1}$ embedding.

Let $X$ be a compact piece of manifold and let $Z$ and $Y$ be boundaryless and orientable $C^{1}$ manifolds of finite dimension, such that $\operatorname{dim} X+\operatorname{dim} Z=\operatorname{dim} Y$ and $Z$ is a closed submanifold of $Y$. A map $f \in C(X, Y)$ is called admissible if $f^{-1}(Z) \subset \operatorname{int}(X)$. Two admissible maps $f_{0}, f_{1} \in C(X, Y)$ are homotopic if there exists $F \in C([0,1] \times X, Y)$ such that $F(s,$.$) is admissible for all s \in[0,1]$, $F(0,)=.f_{0}$ and $F(1,)=.f_{1}$. An admissible map $f$ is transversal if $f$ is $C^{1}$ and, for all $x \in f^{-1}(Z)$, Image $\left(d f_{x}\right)+T_{z}(Z)=T_{z}(Y)$, where $z=f(x)$. As $\operatorname{dim} X+\operatorname{dim} Z=\operatorname{dim} Y$, the sum is direct. The orientation of $T_{x}(X)$ and the map $f$ define a natural orientation of Image $\left(d f_{x}\right)$. If completed with the orientation of $T_{z}(Z)$, it gives the orientation of $T_{z}(Y)$, then the orientation number at $x \in f^{-1}(Z)$ is +1 , and -1 otherwise. Any admissible map $f$ is homotopic to a transversal one $g$ that can be chosen as closed to $f$ in $C(X, Y)$ as necessary. The intersection number of an admissible map $f$ and $Z$ is an integer $I(f, Z) \in \mathbb{Z}$ which satisfies the following properties.

(1) If $f$ is transversal, then $f^{-1}(Z)$ is a finite collection of points and $I(f, Z)$ is the sum of the orientation numbers over $x \in f^{-1}(Z)$.

(2) If $f_{0}$ and $f_{1}$ are homotopic, then $I\left(f_{0}, Z\right)=I\left(f_{1}, Z\right)$.

(3) If $I(f, Z) \neq 0$, then $f^{-1}(Z) \neq \emptyset$.

(4) If $\beta \in C^{1}\left(Y, Y^{\prime}\right)$ is a diffeomorphism, then $I(\beta \circ f, \beta(Z))= \pm I(f, Z)$, depending whether $\beta$ preserves orientation or not.

(5) Let $X_{i}, Z_{i}, Y_{i}, f_{i}$ be admissible for intersection theory $(1 \leqslant i \leqslant k)$. Then $X_{1} \times \cdots \times X_{k}, Z_{1} \times \cdots \times Z_{k}, Y_{1} \times \cdots \times Y_{k}, f_{1} \times \ldots f_{k}$ are also admissible and

$$
I\left(f_{1} \times \cdots \times f_{k}, Z_{1} \times \cdots \times Z_{k}\right)=\prod_{i=1}^{k} I\left(f_{i}, Z_{i}\right) .
$$

Let $X_{1}$ and $X_{2}$ be compact pieces of manifolds and let $Y$ be an orientable $C^{1}$ manifold of finite dimension such that $\operatorname{dim} X_{1}+\operatorname{dim} X_{2}=\operatorname{dim} Y$. A pair of maps $f_{i} \in C\left(X_{i}, Y\right), i=1,2$, is called admissible if $f_{1}\left(x_{1}\right)=f_{2}\left(x_{2}\right)$ implies $x_{1} \in \operatorname{int}\left(X_{1}\right)$ and $x_{2} \in \operatorname{int}\left(X_{2}\right)$. In such a case, the intersection number of $f_{1}$ and $f_{2}$ is defined by

$$
I\left(f_{1}, f_{2}\right)=(-1)^{\operatorname{dim} X_{2}} I\left(f_{1} \times f_{2}, \Delta\right),
$$

$\Delta$ denoting the diagonal in $Y^{2}$ endowed with the orientation induced from the one of $Y$. The factor $(-1)^{\operatorname{dim} X_{2}}$ ensures that if $X_{2}$ is a compact submanifold of $Y$ and $f_{2}$ is the inclusion mapping, then the relation $I\left(f_{1}, f_{2}\right)=I\left(f_{1}, X_{2}\right)$ holds. 
When $M_{1}$ and $M_{2}$ are two compact pieces of manifolds included in $Y$ such that the inclusion mappings $f_{1}: M_{1} \rightarrow Y$ and $f_{2}: M_{2} \rightarrow Y$ are $C^{1}$ embeddings, the notation

$$
I\left(M_{1}, M_{2}\right):=I\left(f_{1}, f_{2}\right)
$$

is used. The assumption that $\left(f_{1}, f_{2}\right)$ is an admissible pair amounts to

$$
\operatorname{dim} M_{1}+\operatorname{dim} M_{2}=\operatorname{dim} Y
$$

and $M_{1} \cap M_{2} \subset \operatorname{int}\left(M_{1}\right) \cap \operatorname{int}\left(M_{2}\right)$. Note that the sign of $I\left(M_{1}, M_{2}\right)$ depends on the choice of orientations of $M_{1}$ and $M_{2}$. We mean by topological transversality of $M_{1}$ and $M_{2}$ that $I\left(M_{1}, M_{2}\right) \neq 0$ if $M_{1} \cap M_{2} \neq \emptyset$. To distinguish topological transversality from the classical notion of transversality, we sometimes call the latter algebraic transversality.

An introduction to intersection theory is provided by [18] and [20, ch. 5]. There, the manifolds $X, Y, Z$ are smooth, $X$ is a compact and boundaryless manifold, and the maps can be supposed to be continuous only (see, for example, [20]). By a theorem of Whitney (see, for example, [20, ch. 2, theorem 2.14]), any $C^{1}$ manifold of dimension $m$ can be $C^{1}$ embedded in $\mathbb{R}^{2 m+1}$ as a closed $C^{\infty}$ submanifold. This allows one to deal with $C^{1}$ manifolds. Finally, the theory extends to compact pieces of manifolds because, by our definition of admissible maps, boundaries are not involved in intersections.

\section{References}

1 U. Bessi. Homoclinic and period-doubling bifurcations for damped systems. Ann. Inst. Henri Poincaré, Analyse nonlin. 12 (1995), 1-25.

2 S. Bolotin. Variational criteria for nonintegrability and chaos in Hamiltonian systems. In Hamiltonian mechanics (Toruń, 1993), pp. 173-179. NATO Adv. Sci. Inst. Ser. B Phys., 331 (New York: Plenum, 1994).

3 B. Buffoni. Infinitely many large amplitude homoclinic orbits for a class of autonomous Hamiltonian systems. J. Diff. Eqns 121 (1995), 109-120.

4 B. Buffoni and É. Séré. A global condition for quasi-random behaviour in a class of conservative systems. Commun. Pure Appl. Math. 49 (1996), 285-305.

5 B. Buffoni and J. F. Toland. Global bifurcation of homoclinic and periodic orbits for autonomous Hamiltonian systems. J. Diff. Eqns 118 (1995), 104-120.

6 B. Buffoni, A. R. Champneys and J. F. Toland. Bifurcation and coalescence of a plethora of multi-modal homoclinic orbits for a Hamiltonian system. J. Dyn. Diff. Eqns 8 (1996), 221-281.

7 B. Buffoni, M. Groves and J. F. Toland. A plethora of capillary-gravity waves with nearcritical Bond and Froude numbers. Phil. Trans. R. Soc. Lond. A 354 (1996), 575-607.

8 A. R. Champneys and A. Spence. Hunting for homoclinic orbits in reversible systems: a shooting technique. Adv. Comp. Math. 1, 81-108.

9 A. R. Champneys and J. F. Toland. Bifurcation of a plethora of multi-modal homoclinic orbits for autonomous Hamiltonian systems. Nonlinearity 6 (1993), 665-721.

10 R. C. Churchill and D. L. Rod. Pathology in dynamical systems. I. J. Diff. Eqns 21 (1976), 3965

11 R. C. Churchill and D. L. Rod. Pathology in dynamical systems. II. J. Diff. Eqns 21 (1976), 66-112.

12 R. C. Churchill and D. L. Rod. Pathology in dynamical systems. III. J. Diff. Eqns 37 (1979), 23-39.

13 V. Coti Zelati and P. H. Rabinowitz. Homoclinic type solutions for a semilinear elliptic PDE on $R^{n}$. Commun. Pure Appl. Math. 45 (1992), 1217-1269.

14 R. L. Devaney. Homoclinic orbits in Hamiltonian systems. J. Diff. Eqns 21 (1976), 431-438. 
R. W. Easton. Isolating blocks and symbolic dynamics. Journal of Diff. Eqns 17 (1975), $96-118$.

16 I. Fonseca and W. Gangbo. Degree theory in analysis and applications (Oxford University Press, 1995).

17 J. Guckenheimer and P. Holmes. Nonlinear oscillations. Dynamical Systems and Bifurcations of Vector Fields (Springer, 1983).

18 V. Guillemin and A. Pollack. Differential topology (Englewood Cliffs, NJ: Prentice-Hall, 1974).

19 S. P. Hastings. Use of simple shooting to obtain chaos. Physica D 62 (1993), 87-93.

20 M. W. Hirsch. Differential topology (Springer, 1976).

21 H. Hofer and J. F. Toland. Homoclinic, heteroclinic and periodic orbits for a class of indefinite Hamiltonian systems. Math. Annalen 268 (1984), 387-403.

W. D. Kalies and R. C. A. M. van der Vorst. Multitransition homoclinic and heteroclinic solutions of the extended Fisher-Kolmogorov equation. J. Diff. Eqns 131 (1996), 209-228. W. D. Kalies, J. Kwapisz and R. C. A. M. van der Vorst. Homotopy classes for stable connections between Hamiltonian saddle-focus equilibria. Commun. Math. Phys. 193 (1998), $337-371$.

K. Mischaikov and M. Mrozek. Chaos in the Lorenz equations: a computer-assisted proof. Bull. AMS 32 (1995), 66-72.

K. Mischaikov and M. Mrozek. Isolating Neighbourhoods and chaos. Japan J. Ind. Appl. Math. 12 (1995), 205-236.

K. Mischaikov and M. Mrozek. Chaos in the Lorenz equations: a computer-assisted proof (part II: details). Math. Comput. 67 (1998), 1023-1046.

K. J. Palmer. Exponential dichotomies, the shadowing lemma and transversal homoclinic points. Dyn. Rep. 1 (1988), 265-306.

28 J. Palis and W. de Melo. Geometric theory of dynamical systems (Springer, 1982).

29 L. A. Peletier and W. C. Troy. Spatial patterns described by the extended FisherKolmogorov equation: kinks. Differential Int. Equations 8 (1995), 1279-1304.

30 L. A. Peletier and W. C. Troy. A topological shooting method and the existence of kinks of the extended Fisher-Kolmogorov. Topolog. Methods Nonlinear Analysis 6 (1995), 331-355. L. A. Peletier and W. C. Troy. Chaotic spatial patterns described by the extended FisherKolmogorov equation. J. Diff. Eqns 129 (1996), 458-508.

É. Séré. Looking for the Bernoulli shift. Ann. Inst. Henri Poincaré, Analyse nonlin. 10 (1993), 561-590.

33 J. F. Toland. Hamiltonian systems with monotone trajectories. Proc. Centre Math. Analysis Aust. Nat. Univ. 8 (1984), 51-63.

34 J. F. Toland. An index for Hamiltonian systems with a natural order structure. In Nonlinear functional analysis and its applications (Maratea, 1985), pp. 147-160. NATO Adv. Sci. Inst. Ser. C Math. Phys. Sci, 173 (Dordrecht and Boston, MA: Reidel, 1986).

J. B. van den Berg. Uniqueness of solutions for the extended Fisher-Kolmogorov equation.

C. R. Acad. Sci. Paris, Sér. I Math. 326 (1998), 447-452.

(Issued 3 December 1999) 